\title{
Hybrid Dual-Hop RF/FSO Terrestrial-Deep Space Communication System under Solar Scintillation during Superior Solar Conjunction
}

\author{
Meng Jin ${ }^{1,2}$, Wenyi Liu ${ }^{1,2}$, Yuan Hao ${ }^{1,2}$, Ruihuan $W u^{1,2, *}$, Zhongchao Wei ${ }^{1,2} \mathbb{D}$, Dongmei Deng ${ }^{1,2}$ \\ and Hongzhan Liu 1,2,*(D) \\ 1 Guangdong Provincial Key Laboratory of Nanophotonic Functional Materials and Devices, \\ Guangzhou 510006, China; jinm@m.scnu.edu.cn (M.J.); wyliu@m.scnu.edu.cn (W.L.); \\ 2018022081@m.scnu.edu.cn (Y.H.); wzc@scnu.edu.cn (Z.W.); dmdeng@263.net (D.D.) \\ 2 School of Information and Optoelectronic Science and Engineering, South China Normal University, \\ Guangzhou 510006, China \\ * Correspondence: wurh@scnu.edu.cn (R.W.); lhzscnu@163.com (H.L.)
}

check for

updates

Citation: Jin, M.; Liu, W.; Hao, Y.; Wu, R.; Wei, Z.; Deng, D.; Liu, H. Hybrid Dual-Hop RF/FSO Terrestrial-Deep Space Communication System under Solar Scintillation during Superior Solar Conjunction. Appl. Sci. 2022, 12, 619. https://doi.org/10.3390/ app12020619

Academic Editors: Shuangyi Yan and Hui Yang

Received: 29 November 2021

Accepted: 4 January 2022

Published: 10 January 2022

Publisher's Note: MDPI stays neutral with regard to jurisdictional claims in published maps and institutional affiliations.

Copyright: (c) 2022 by the authors. Licensee MDPI, Basel, Switzerland. This article is an open access article distributed under the terms and conditions of the Creative Commons Attribution (CC BY) license (https:/ / creativecommons.org/licenses/by/ $4.0 /)$.

\begin{abstract}
Free-space optical communication (FSO) technology has wide prospects in deep space exploration, but it will encounter coronal turbulence during superior solar conjunction, and solar scintillation will seriously affect the communication quality. In this paper, we propose a terrestrialdeep space hybrid radio frequency (RF)/FSO system with the hybrid L-pulse position modulationbinary phase shift keying-subcarrier intensity modulation (L-PPM-BPSK-SIM) scheme, where the RF channel of the satellite-terrestrial relay follows the Rayleigh distribution, and the FSO channel of the relay satellite to the deep space probe adopts Gamma-Gamma distribution. Considering the pointing error, the expression of the bit error rate (BER), the outage probability, and the average channel capacity of the hybrid system are derived. In addition, we evaluated the influence of coronal turbulence parameters on the system through amplitude fluctuations. The simulation results demonstrate that the hybrid RF/FSO system improves the BER performance by 10 to 30 times in a deep space environment, and the use of a hybrid modulation can further reduce the BER. The nonKolmogorov spectral index, outer scale, solar wind density fluctuation factor, and optical wavelength comprehensively affect the BER through amplitude fluctuations. Our research results have potential application value for evaluating the link performance of future deep space communications.
\end{abstract}

Keywords: deep space communication; RF/FSO system; solar scintillation; PPM-BPSK-SIM; bit error rate; pointing error

\section{Introduction}

With the development of deep space exploration, improving the communication performance of deep space links has become a challenging topic. Free space optical (FSO) is a viable solution thanks to its advantages of high data transmission and large capacity $[1,2]$. However, the communication link will face great challenges during superior solar conjunction when the Sun is located between the Earth and the probe [3]. At this time, due to the random changes in the refractive index of the coronal turbulence, the FSO link encounters the irregular solar wind plasma from the Sun, resulting in dramatic signal fluctuations. This phenomenon is called solar scintillation [4]. The essence of solar scintillation is wave scattering, which is directly related to signal amplitude fluctuations, phase fluctuations and angle of arrival fluctuations. The impact of coronal turbulence on the Earth-detector link has been reported in the astronomy and communication fields [5,6], and will lead to the performance of the communication links degrading severely, and may even cause the interruption. Xu et al. derived the closed-form amplitude fluctuation expression of optical waves propagating in non-Kolmogorov solar wind turbulence during superior solar 
conjunction, for the first time, based on the Rytov approximation, and briefly discussed the bit error rate (BER) of the FSO system under the log-normal (LN) channel [7]. Then, a further study uses M-ary PPM modulation to improve the BER and average channel capacity in the deep space FSO communication under the LN channel [8]. So far, most of the research in deep space optical communications have focused on FSO communication systems. However, deep space communications are affected by solar activities, and irregular solar wind over long distances will cause signal distortion and fading. In addition, the atmosphere around the earth will also produce signal attenuation. Therefore, further enhancement in the performance of FSO system is required.

In near-Earth communication, the combination of FSO technology and RF technology has become a complementary communication technology to alleviate the interference of atmospheric turbulence [9]. As a dual-hop relay system with asymmetric channels, the hybrid RF/FSO relay system combines the advantages of RF systems and FSO systems. Nowadays, there have been numerous studies on hybrid RF/FSO systems [9-11]. The authors in [10] discussed an RF/FSO system, obeying the Nakagami-m/Gamma-Gamma distribution, derived the end-to-end probability density function (PDF) expression, and simulated the BER and channel capacity performance. Based on the Rayleigh/Gamma-Gamma model proposed by [9], the outage probability, the average BER and channel capacity of the system are comprehensively studied [11]. This model is robust in the simulation of turbulence at different intensities and has been recognized by many researchers.

The theoretical study of the hybrid RF/FSO system is well established and it has been proven to perform better than the FSO system in various scenarios. In $[12,13]$, the theory of 2-hop downlink in deep space communications is discussed, and high link availability is demonstrated. Some papers successively suggested that the hybrid system can be applied to deep space communication [1-3,14-17]. In [14,15], it was the first time that an RF-Optical method was proposed to improve the performance of deep space communications. A reasonable and promising scheme of the FSO/RF system was introduced in [2], in which the optical signal was first transmitted from the deep space detector to the geostationary orbit satellite through FSO, and then to the receiver on the Earth via RF. After that, Xu proposed that a hybrid RF/FSO system could be used to mitigate the signal attenuation caused by solar scintillation [3]. Recently, considering the channel state information (CSI), the outage probability of the hybrid RF/FSO deep space-terrestrial system based on the Rician/Gamma-Gamma distribution was studied [16]. To the best of our knowledge, this is the first detailed theoretical research on the introduction of RF/FSO into deep space communications. Subsequently, Liu et al. applied an empirical scintillation model to study the BER performance of the hybrid RF/FSO deep space communications system [17].

Inspired by the above researches, this paper applies the hybrid RF/FSO system to deep space communication based on the scintillation model proposed in [7], and discusses the end-to-end communication performance. We propose a terrestrial-deep space hybrid RF/FSO system using hybrid L-pulse position modulation-binary phase shift keyingsubcarrier intensity modulation (L-PPM-BPSK-SIM) scheme, where the RF link obeys Rayleigh fading, and the FSO link follows Gamma-Gamma distribution. When discussing the channel model, so as to obtain the relationship between solar scintillation and BER more accurately, we neglected the attenuation factor of the troposphere. Then considering the influence of the coronal turbulence during the superior solar conjunction period, the background model of the non-Kolmogorov spectrum solar wind turbulence was introduced in detail through the weak electromagnetic scintillation theory, and the research on amplitude fluctuations was conducted. As far as we know, there have been many studies on the influence of solar scintillation on radio wave communications [18-20], but studies for the hybrid RF/FSO system are relatively rare. On this basis, considering the pointing error, the outage probability, BER, and average channel capacity expressions of the hybrid RF/FSO system in the deep space are derived. According to the closed analytical formula, we further analyzed the effects of the modulation scheme, coronal parameters, and system parameters on solar scintillation and system performance. 
The rest of this paper is arranged as follows: Section 2 introduces the amplitude scintillation theory based on the non-Kolmogorov coronal turbulence model, the RF/FSO hybrid system communication model and hybrid L-PPM-BPSK-SIM scheme. Section 3 further deduces the formula of outage probability, BER, and average channel capacity of the system. Then in Section 4, the theoretical results are simulated and discussed. Finally, Section 5 provides some concluding remarks.

\section{System and Theoretical Model}

In order to analyze the impact of coronal plasma on optical links, the Figure 1 shows the geometric diagram for deep space communication during superior conjunction. Here, the distance between the Earth and the Sun is $L_{s e}$ and the distance between the probe and the Sun is $L_{s p}$. Note that, $z$ denotes the signal propagation distance, $r$ is the distance between the Sun and the communication link, which is usually in units of the solar radius $R_{\text {sun }}$. Therefore, both the Sun-Earth-Probe (SEP) angle $\alpha$ and the Sun-Probe-Earth (SPE) angle $\beta$ are directly related to $r$. According to the triangle relationship, the link distance can be written as:

$$
L_{\text {link }}=L_{s e} \cos \alpha+L_{s p} \cos \beta \text {. }
$$

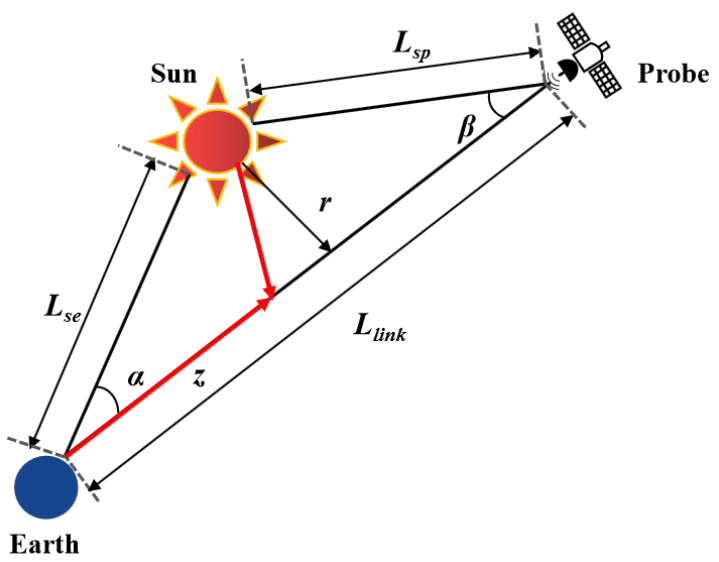

Figure 1. Geometric diagram for deep space communication during superior conjunction.

\subsection{Amplitude Fluctuations Variance Model}

Solar wind erupts from the Sun stochastically, and it fluctuates in space and time. The study of solar wind belongs to the field of space physics. Exploring solar wind plasma theory is not only related to human understanding of physical processes, such as stellar particle acceleration, stellar energy transport, space weather change, and magnetic reconnection, but it is also related to ensuring the safety of human outer space and the normal communication between deep space detectors and earth observation stations. In recent years, electromagnetic studies on optical signal propagation have been concerned by researchers [21-23]. As the main factor affecting the relationship between the Sun and the Earth, the research on the variation of solar wind has gradually attracted the attention of space physics and received extensive research.

The accurate solar wind model does not yet exist. However, the spatial spectrum of solar wind can be used to describe the irregularity of its turbulence. When ignoring coronal mass ejections, the turbulent vortices of solar wind plasma in a certain region is considered to be locally uniform. The observed data indicates that the changes in the internal structure of solar wind turbulence is not pronounced within a wave period, and the turbulence at this time is usually regarded as "frozen", so that "Taylor's freezing theory" might be applied [24]. According to this theory, the coronal turbulence is regarded as a locally homogeneous frozen random medium moving at a constant solar wind velocity. The hypothesis is reasonable and has been widely used in radio astronomy research [25]. Moreover, based on long-term observations, the coronal spectrum has been proved to be 
close to the non-Kolmogorov spectrum [26]. Therefore, we set the value of the spectral index $p$ as $3<p<4$, and then the spatial power spectrum of the density fluctuations in corona is characterized by the generalized von Karman turbulence spectrum and the generalized exponential spectrum as:

$$
\Phi_{N}(\kappa, z)=C_{N}^{2}(z) \kappa^{-p}, \kappa_{i}<\kappa<\kappa_{0},
$$

where $C_{N}^{2}$ is the structural constant of the solar wind coronal turbulence, which is obtained from [25] as follows:

$$
C_{N}^{2}(z)=\left\{\begin{array}{c}
(2 \pi)^{(-3 / 2)} \frac{(p-3) \Gamma\left(\frac{p}{2}\right) \kappa_{o}^{(p-3)}}{\Gamma\left(\frac{p-1}{2}\right)} \delta\left\langle N_{e}(r)\right\rangle_{,}^{2} \text { for } 3<p<4 \\
4 \pi \ln \left(\frac{2 \kappa_{o}}{\kappa_{i}}\right) \delta\left\langle N_{e}(r)\right\rangle^{2}, \text { for } p=3
\end{array},\right.
$$

where $\kappa_{o}=2 \pi / l_{o}, \kappa_{i}=2 \pi / l_{i} . l_{o}$ and $l_{i}$ are the outer and inner scales of the coronal turbulence, respectively. $\Gamma(\cdot)$ is the Gamma function. Using the empirical formula in [27] for the solar wind plasma density, the electron density is obtained as a function of the heliocentric distance $r$ :

$$
N_{e}(r)=2.21 \times 10^{14}\left(\frac{r}{R_{\text {sun }}}\right)^{-6}+1.55 \times 10^{12}\left(\frac{r}{R_{\text {sun }}}\right)^{-2.3} .
$$

Moreover, the irregularities of solar wind plasma density are usually assumed proportional to the plasma density as $\delta N_{e}(r)=\eta N_{e}(r)$, and $\eta$ is solar wind plasma density relative fluctuation factor [25].

As we discussed in Section 1, when the optical signal approaches the solar region, it is scattered by the turbulent medium in the Fresnel region, which results in the amplitude and phase fluctuation of the electromagnetic wave through the solar wind turbulent vortex in the coronal region. These fluctuations are commonly referred to as solar scintillation [28]. Here we only consider the effect of solar scintillation on the amplitude fluctuations of optical waves. Since the amplitude fluctuations usually distribute around their mean levels, the variance of the signal $\chi^{2}$ is an important parameter to characterize its variation. In the weak fluctuation region, the logarithmic amplitude fluctuations propagated by the coronal solar wind turbulence can be obtained according to the weak electromagnetic scintillation theory so that the Rytov theory applies [29]. As a consequence, we have that

$$
\left\langle\chi^{2}\right\rangle=16 \pi^{4} r_{e}{ }^{2} k_{n}^{-2} \int_{0}^{\infty} \kappa d \kappa \int_{0}^{\infty} d z \sin ^{2}\left(\frac{z \kappa^{2}}{2 k_{n}}\right) \Phi_{N}(\kappa, z),
$$

where $\langle\cdot\rangle$ represents the ensemble average of realizations, $k_{n}=2 \pi / \lambda$ is wavenumber, and $\lambda$ denotes the wavelength of the signal. Substituting (2) into (5), the final simplified amplitude fluctuation variance model is acquired after some numerical transformations [7]:

$$
\left\langle\chi^{2}\right\rangle=-\frac{(p-3) \Gamma\left(\frac{p}{2}\right) r_{e}^{2}(2 \pi)^{p-1 / 2} \pi}{8 \Gamma\left(\frac{p-1}{2}\right) \Gamma\left(1+\frac{p}{2}\right)} \times \eta^{2} N_{e}{ }^{2} l_{o}^{3-p} L_{l i n k}{ }^{\frac{p}{2}} k_{n}^{-\frac{p}{2}-1} \sec \left(\frac{\pi}{4} p\right)
$$

where $r_{e}$ is the classical electron radius $\left(=2.8 \times 10^{-15} \mathrm{~m}\right)$. In addition, scintillation index $\mathrm{m}^{2}$ is another parameter for estimating amplitude fluctuation, which is defined as the ratio of the root mean square value of signal strength fluctuations to the mean signal intensity:

$$
m^{2}=\frac{\left\langle(I-\langle I\rangle)^{2}\right\rangle}{\langle I\rangle^{2}}, m \leq 1,
$$

where $I$ is the received intensity. Additionally, many studies have proved that $m^{2}=4\left\langle\chi^{2}\right\rangle$ [29]. According to the magnitude of scintillation index, we can divide it into weak scintillation 
and strong scintillation. It is generally accepted that a scintillation index less than 0.3 generates a weak scintillation effect, an index between 0.3 and saturation indicates the transition region, and strong scintillation is determined by an index of 1 . Since the derived scintillation index model is based on Rytov theory, that is, under the premise of weak scintillation, we have that $m^{2}<1$.

\subsection{Hybrid RF/FSO System Model}

Figure 2 demonstrates the asymmetric-based RF/FSO deep space communication system during superior conjunction. We adopt the dual-hop relay communication technology, where relay satellites orbiting the Earth first receive signals from Earth stations through RF and then transmit them via FSO.

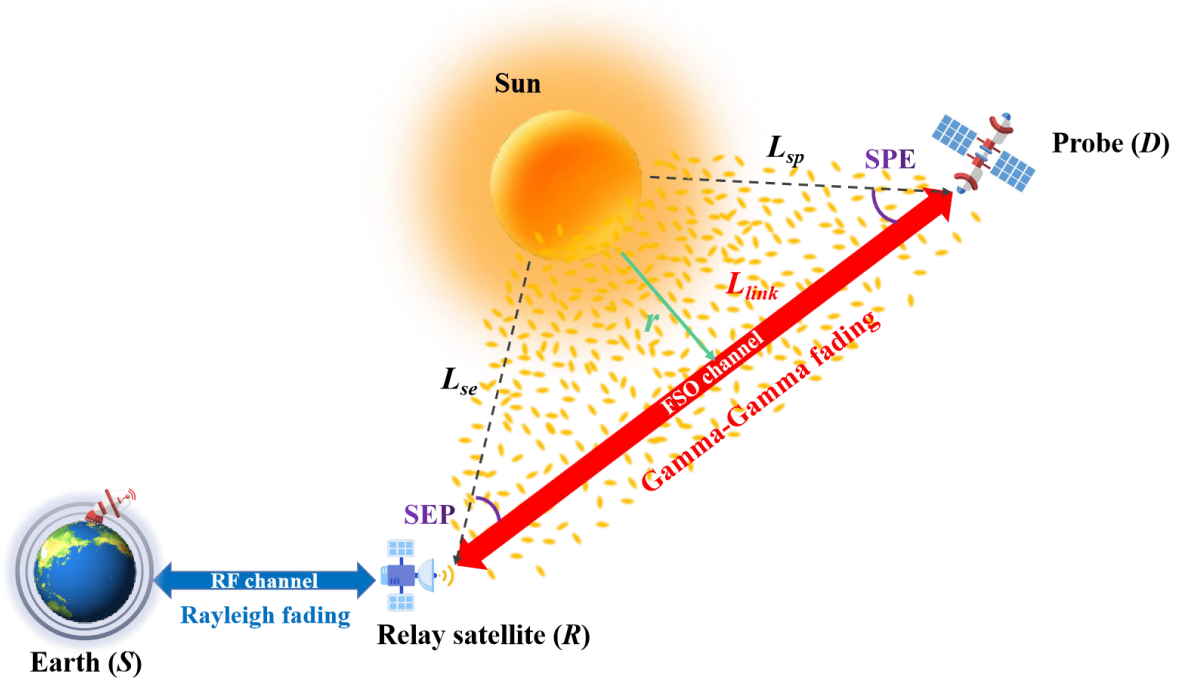

Figure 2. Diagram of deep space RF/FSO communication system.

The RF link in the hybrid system obeys the Rayleigh distribution, and its probability density function (PDF) is expressed as:

$$
f_{\gamma_{1}}\left(\gamma_{1}\right)=\frac{1}{\overline{\gamma_{1}}} \exp \left(-\frac{\gamma_{1}}{\overline{\gamma_{1}}}\right)
$$

where $\gamma_{1}$ represents the instantaneous signal-to-noise ratio (SNR) of the RF link, and $\overline{\gamma_{1}}$ represents the average SNR of the RF channel. Therefore, the CDF subject to Rayleigh distribution can be written as:

$$
F_{1}\left(\gamma_{1}\right)=1-\exp \left(-\frac{\gamma_{1}}{\overline{\gamma_{1}}}\right)
$$

On the other hand, in order to describe the weak to strong turbulence more accurately, we use the Gamma-Gamma distribution to describe the FSO channel fading while considering the pointing error. Thus, the PDF of the SNR is expressed as [11]:

$$
f_{\gamma_{2}}\left(\gamma_{2}\right)=\frac{\xi^{2}}{2 \gamma_{2} \Gamma(\varphi) \Gamma(\phi)} G_{1,3}^{3,0}\left[\varphi \phi \sqrt{\frac{\gamma_{2}}{\overline{\gamma_{2}}}} \mid \begin{array}{c}
\xi^{2}+1 \\
\xi^{2}, \varphi, \phi
\end{array}\right],
$$

where $\gamma_{2}$ represents the instantaneous SNR of the FSO link and $\overline{\gamma_{2}}$ represents the average SNR of the FSO link. $\xi=\frac{\omega_{e}}{2 \sigma_{s}}$ is a pointing error parameter, which denotes the ratio of the equivalent beam waist radius and jitter standard deviation at the receiving end. $G(\cdot)$ is the Meijer's G function as defined in [30]. $\varphi$ and $\phi$ are the large-scale scattering coefficient and the small-scale scattering coefficient, respectively, which are related to the beam type. For spherical waves, they can be expressed as [31]: 


$$
\begin{gathered}
\varphi=\left[\exp \left(\frac{0.49 \sigma_{l}^{2}}{\left(1+0.18 d^{2}+0.56 \sigma_{l}^{12 / 5}\right)^{7 / 6}}\right)-1\right]^{-1}, \\
\phi=\left[\exp \left(\frac{0.51 \sigma_{l}^{2}\left(1+0.69 \sigma_{l}^{12 / 5}\right)^{-5 / 6}}{\left(1+0.9 d^{2}+0.62 d^{2} \sigma_{l}{ }^{12 / 5}\right)^{5 / 6}}\right)-1\right]^{-1},
\end{gathered}
$$

where $\sigma_{l}^{2}$ is the logarithmic intensity fluctuation variance, $d=\left(k_{n} D_{m}{ }^{2} / 4 L_{\text {link }}\right)^{1 / 2}$, and $D_{m}$ is the receiver aperture. Jokippi et al. proved that the variance of logarithmic intensity fluctuations and the variance of logarithmic amplitude fluctuations under weak turbulence are as follows [32]:

$$
\sigma_{l}^{2}=4\left\langle\chi^{2}\right\rangle
$$

Therefore, the scintillation index for the Gamma-Gamma channel model is further written as:

$$
m^{2}=\frac{1}{\varphi}+\frac{1}{\phi}+\frac{1}{\varphi \phi} .
$$

Considering the complexity of the system, a fixed-gain amplify-and-forward (AF) relay technology is adopted, while an intensity modulation/direct detection (IM/DD) technique is used at the receiving end. Therefore, the end-to-end SNR can be expressed as $\gamma=\frac{\gamma_{1} \gamma_{2}}{\gamma_{2}+C}$ (Appendix A), where $C$ is the fixed relay gain.

The CDF of the hybrid RF/FSO system is expressed as [33]:

$$
F_{\gamma}(\gamma)=1-A_{1} \sqrt{\gamma} \exp \left(-\frac{\gamma}{\overline{\gamma_{1}}}\right) \times G_{1,6}^{6,0}\left[B \gamma \mid \begin{array}{c}
\frac{\xi^{2}+1}{2} \\
o_{1}
\end{array}\right]
$$

where $A_{1}=\frac{2^{(\varphi+\phi)} \varphi \phi \xi^{2} \sqrt{C}}{32 \pi \sqrt{\overline{\gamma_{1}}} \sqrt{\bar{\gamma}_{2}} \Gamma(\varphi) \Gamma(\phi)}, B=\frac{(\varphi \phi)^{2} C}{16 \bar{\gamma}_{1} \gamma_{2}}, o_{1}=\frac{\tilde{\xi}^{2}-1}{2}, \frac{\varphi-1}{2}, \frac{\varphi}{2}, \frac{\phi-1}{2}, \frac{\phi}{2},-\frac{1}{2}$.

\subsection{Hybrid PPM-BPSK-SIM Modulation Scheme}

A hybrid PPM-BPSK-SIM modulation is a multi-dimensional modulation method that combines subcarrier modulation and pulse position modulation. Here, we introduce by a 4-PPM, which is displayed in Figure 3. For a 4-PPM modulation symbol, the number of slots is $L=4$, and the data bits that can be transmitted in each frame $\mathrm{n}=\log _{2} L=2$. If the n-bit data are written as $M$, and the time slot position of the light pulse is recorded as $l, M$ can have four combinations: $M=(0,0), M=(1,0), M=(0,1), M=(1,1)$, and the corresponding time slot positions are $l=0, l=1, l=2, l=3$, respectively. As shown in Figure 3, the L-PPM-BPSK is to change the original position of energy in L-PPM from pure intensity modulation to BPSK modulation. Therefore, after the combination of PPM and BPSK, one bit of BPSK information is added to each frame, and the number of transmitted bits is increased, which improves the transmission efficiency.

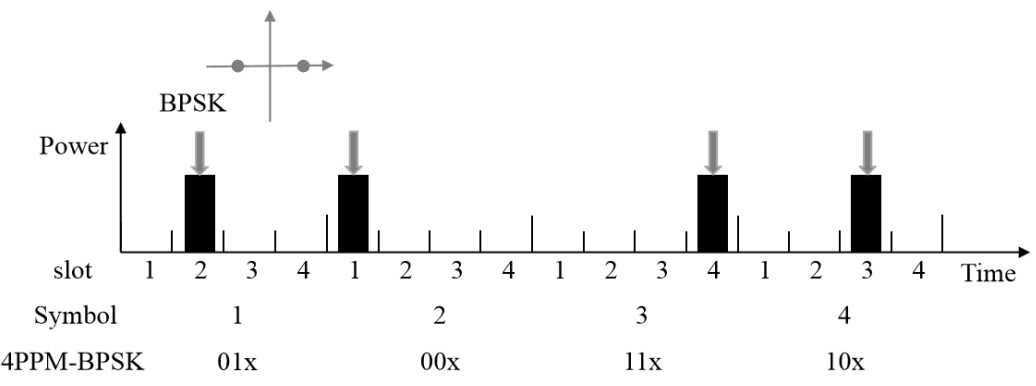

Figure 3. 4-PPM-BPSK modulation schematic diagram. 
As present in Figure 4, the transmitter consists of an n-bit PPM encoder, a parallel serial converter and a transmitter filter with a rectangular pulse shape of duration. The PPM symbol is applied to the BPSK modulator on the radio frequency subcarrier. Before intensity modulation of the light source, a DC bias is added to the BPSK signal. Therefore, the modulated signal with L-PPM-BPSK-SIM is expressed as

$$
s(t)=\sum_{k=1}^{L} I_{k}\left[1+\varsigma \cos \left(\omega_{c} t+c_{k} \pi\right) g\left(t-(k-1) T_{s}\right)\right],
$$

where $I_{k}$ is the intensity of the $k$-th code, $\varsigma$ and $\omega_{c}$ represent the optical modulation index and the carrier frequency, respectively. $c_{k} \in\{0,1\}$ is the code word of PPM, $g(t)$ is the pulse shaping function, and $T_{\mathcal{S}}$ denotes the symbol interval [34,35].

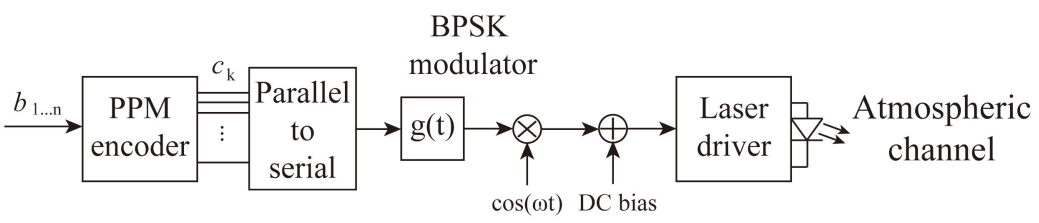

(a)

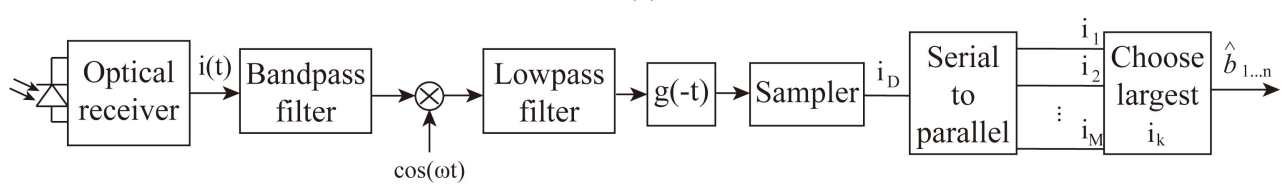

(b)

Figure 4. Block diagram of the hybrid PPM-BPSK-SIM system; (a) transmitter, (b) receiver.

The receiving front end is composed of photodetectors and amplifiers. So its resultant output signal for one symbol stream, which is proportional to the modulated signal, can be obtained as,

$$
r(t)=R \alpha_{c} \sum_{k=1}^{L} I_{k}\left[1+\varsigma \cos \left(\omega_{c} t+c_{k} \pi\right) g\left(t-(k-1) T_{s}\right)\right]+n(t)
$$

where $R$ is photodetector responsivity, $\alpha_{c}$ is channel attenuation and $n(t)$ is total receiver noise.

\section{Performance Analysis}

\subsection{Outage Probability}

Outage probability is defined as the probability that the instantaneous SNR of the system, $\gamma$, is lower than a certain threshold value $\gamma_{t h}$; therefore, the outage probability of a hybrid RF/FSO system is deduced by the definition $P_{\text {out }}\left(\gamma_{t h}\right)=\operatorname{Pr}\left(\gamma<\gamma_{t h}\right)$ :

$$
P_{\text {out }}\left(\gamma_{t h}\right)=F_{\gamma}\left(\gamma_{t h}\right)=1-A_{1} \sqrt{\gamma_{t h}} \exp \left(-\frac{\gamma_{t h}}{\overline{\gamma_{1}}}\right) \times G_{1,6}^{6,0}\left[\begin{array}{c|c}
B \gamma & \frac{\xi^{2}+1}{2} \\
o_{1}
\end{array}\right] \text {. }
$$

\subsection{Average Bit Error Rate}

In Section 2, we specifically introduced the system model. The BER performance is usually closely related to modulation method. There are many modulation methods that can be used in hybrid RF/FSO systems. To our knowledge, the common modulation method in deep space optical communications is PPM, and BPSK with good anti-interference performance is the classical modulation method in the near-Earth hybrid system.

According to $[33,34,36]$, the conditional BER of L-ary pulse position modulation $(L-P P M)$ is:

$$
P_{L P P M}=\frac{1}{2} \operatorname{erfc}\left(\frac{1}{2 \sqrt{2}} \sqrt{\gamma \frac{L}{2} \log _{2} L}\right) \text {. }
$$


By [11], the common unified expression of the conditional BER of binary modulation in Gaussian white noise is

$$
P_{e}(e \mid \gamma)=\frac{\Gamma(a, b \gamma)}{2 \Gamma(b)}
$$

where $a, b$ are modulation parameters, and when $a=0.5, b=1$, it is BPSK modulation.

Base on L-PPM-BPSK-SIM schemes, We further assume an equivalent probable data transmission for both conditional and unconditional BER calculation over the channel model. So, the conditional BER with Gaussian white noise is [33]:

$$
P_{L P P M-B P S K-S I M}=Q\left(\frac{1}{4} \sqrt{\gamma L \log _{2} L}\right),
$$

where $Q(\cdot)$ is the Gaussian $Q$ function.

The unconditional BER of the system affected by the coronal turbulent media is defined as:

$$
P_{B E R}=\int_{0}^{\infty} P_{e}(\gamma) f_{\gamma}(\gamma) d \gamma,
$$

where $P_{e}(\gamma)$ is the conditional BER of modulation mode in Gaussian white noise, and $f_{\gamma}(\gamma)$ is the PDF of the hybrid RF/FSO system, which can be obtained by differentiating (14):

$$
f_{\gamma}(\gamma)=\left(\frac{A_{2}}{\overline{\gamma_{1}}}-\frac{B}{A_{2}}\right) \exp \left(-\frac{\gamma}{\overline{\gamma_{1}}}\right) \times\left(G_{1,6}^{6,0}\left[B \gamma \mid \begin{array}{c}
\frac{\xi^{2}}{2}+1 \\
o_{2}
\end{array}\right]+G_{2,7}^{6,1}\left[B \gamma \mid \begin{array}{c}
o_{3} \\
o_{4}
\end{array}\right]\right),
$$

where $A_{2}=\frac{\xi^{2} 2^{\varphi+\phi}}{8 \pi \Gamma(\varphi) \Gamma(\phi)}, o_{2}=\frac{\xi^{2}}{2}, \frac{\varphi}{2}, \frac{\varphi+1}{2}, \frac{\phi}{2}, \frac{\phi+1}{2}, 0, o_{3}=-1, \frac{\xi^{2}-1}{2}, o_{4}=\frac{\xi^{2}}{2}-1, \frac{\varphi}{2}-1, \frac{\varphi-1}{2}, \frac{\phi}{2}-$ $1, \frac{\phi-1}{2},-1,0$.

Substituting (20) and (22) into (21), the approximate closed-form expression of the unconditional BER of the hybrid RF/FSO system modulated by L-PPM-BPSK-SIM can be obtained:

$$
P_{B E R}=-\frac{1}{2 \sqrt{\pi}} \Gamma\left(\frac{1}{2}\right)+A_{2} \frac{\sqrt{\log _{2} L}}{8 \sqrt{2 \pi}} \times\left(\frac{L \log _{2} L}{32}-\frac{1}{\overline{\gamma_{1}}}\right)^{-\frac{1}{2}} G_{2,6}^{6,1}\left[\frac{B}{\frac{\overline{\gamma_{1} L \log _{2} L}}{32}-1} \mid \begin{array}{c}
o_{5} \\
o_{2}
\end{array}\right],
$$

where $o_{5}=\frac{1}{2}, \frac{\xi^{2}}{2}+1$

\subsection{Average Channel Capacity}

The Shannon channel capacity is essentially time-varying relative to the instantaneous value of the received SNR. Therefore, average channel capacity is often used for evaluation. The precise definition of the average channel capacity is obtained by [37], $\bar{C}=E\left[B \log _{2}(1+\gamma)\right]$, where $E(\cdot)$ refers to the mathematical expectation operator. Usually, we deduce the average channel capacity using the Shannon formula modified by channel CDF:

$$
\bar{C}=\frac{1}{\ln 2} \int_{0}^{\infty} \frac{1-F_{\gamma}(\gamma)}{1+\gamma} d \gamma
$$

Utilizing $\frac{1}{1+x}=G_{0,0}^{1,1}\left(x \mid \begin{array}{l}0 \\ 0\end{array}\right)$, substituting (14) into (24) and using the extended generalized bivariate Meijer's G function (EGBMGF) in [38], we obtain the average channel capacity as:

$$
\bar{C}=\frac{2^{(\varphi+\phi)} A_{1} \sqrt{C}}{16 \pi \ln 2} \overline{\gamma_{1}} \times G_{0,0: 0,0: 1,6}^{0,0: 1,1: 6,0}\left[\begin{array}{c|c|c|c}
- & 0 & \frac{\xi^{2}+1}{2} & \mid \overline{\gamma_{1}}, \frac{(\varphi \phi)^{2} C}{16 \overline{\gamma_{2}}}
\end{array}\right]
$$

\section{Simulation and Discussion}

In this section, we numerically evaluate the closed analytical formula obtained in Section 3 to study the performance of a hybrid RF/FSO system modulated by L-PPM-BPSK- 
SIM under the coronal turbulence. We mainly consider the communication between Earth and Mars. Therefore, the distance between the Earth and the probe is set as $L_{s p}=1.5 L_{s e}$, which is suitable for Mars's orbit during superior solar conjunction, where the distance between the Earth and the Sun is $L_{s e}=1.5 \times 10^{8} \mathrm{~km}$. For simplicity, the SPE angle and relative solar wind density fluctuation factor are set as fixed values, $\beta=1.2^{\circ}, \eta=10 \%$, respectively. According to the experience of NASA deep space exploration [3,39], we use a $32 \mathrm{GHz}$ radio frequency and consider the transmission power to be $75 \mathrm{~W}$. In addition, the optical wavelengths of $\lambda=850,1064$, and $1550 \mathrm{~nm}$ are selected. In this paper, unless otherwise specified, the wavelength is selected at $1550 \mathrm{~nm}$, and other parameters used in the calculation are as follows: SEP angle $0.3^{\circ}<\alpha<0.5^{\circ}$, spectral index $p=11 / 3$, outer scale $l_{o}=5 \times 10^{7} \mathrm{~m}$, receiver aperture $D_{m}=0.1 \mathrm{~m}$. At the same time, for the convenience of calculation, the average SNR of RF link and FSO link is equal in simulation, i.e., $\overline{\gamma_{1}}=\overline{\gamma_{2}}=\bar{\gamma}$, and the gain constant at the relay is assumed to be $C=1$ [40].

Figure 5 describes the simulation results of the relationship between SEP angle and scintillation index at different wavelengths. The results indicate that as the SEP angle increases, the scintillation index also increases. The SEP angle will directly affect the heliocentric distance and the smaller the SEP angle, the closer the communication link to the Sun. Therefore, the link will encounter stronger coronal turbulence and the amplitude fluctuation caused by solar scintillation become more severe. In addition, signals with larger wavelengths are more susceptible to coronal turbulence. This phenomenon is reasonable because the spatial coherence length is proportional to the wavelength. Therefore, a smaller wavelength may be considered as an option to improve the quality of deep-space optical communications.

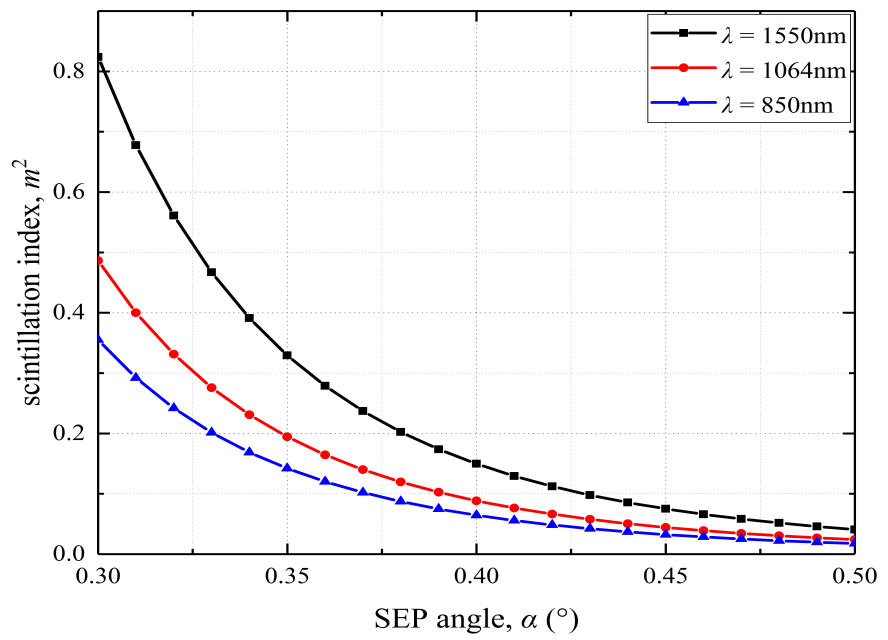

Figure 5. The scintillation index against wavelength under different SEP angles.

Figure 6 depicts the outage probability performance with the scintillation index given by $0.1,0.3$, and 0.5 , respectively. As the scintillation index increases, the outage probability also increases. As the overall range is set within the weak scintillation, the increase is not great. As the scintillation boosting, the outage probability may increase significantly and even lead to complete outage of the system. Additionally, the influence of the pointing error is considered. The pointing error of the system will have different effects on the interruption probability under different turbulence intensities. With the increase of the pointing error parameter $\xi$, the outage probability has been significantly reduced. For example, when average SNR is $15 \mathrm{~dB}, m^{2}=0.1$, the outage probability of $\xi=1$ is $4.798 \times 10^{-1}$, while the outage probability of the $\xi=5$ is $3.439 \times 10^{-1}$. Consistent with our theoretical description, the impact of the pointing error on the system will be smaller as $\xi$ grows larger. 


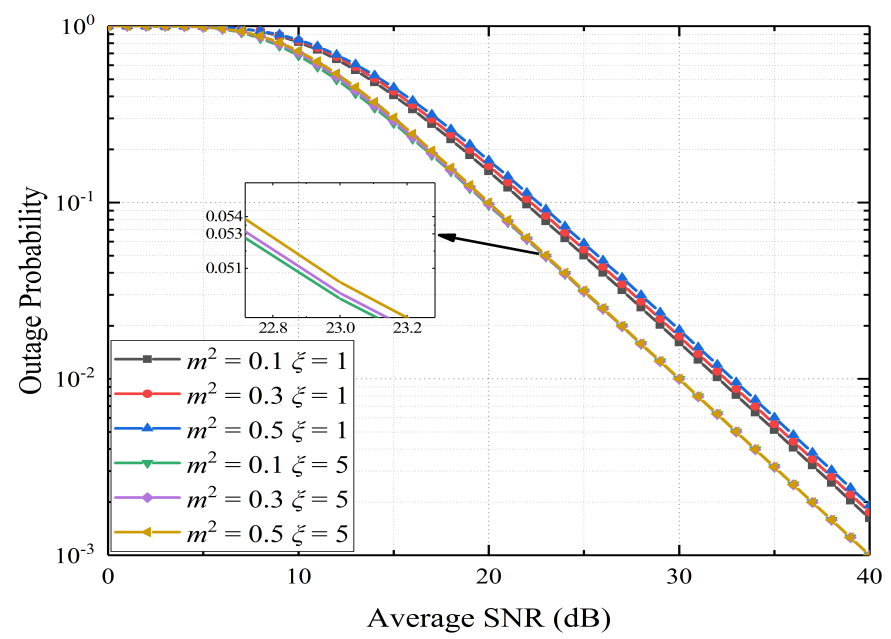

Figure 6. Outage probability against average signal-to-noise ratio (SNR) per hop for various scintillation intensities and different pointing error parameters. The threshold of the hybrid system is $\gamma_{t h}=10 \mathrm{~dB}$.

To observe the effect of coronal turbulence on the average BER performance of pure FSO and hybrid RF/FSO systems, we compare two systems by the same modulation technology (16-PPM-BPSK-SIM). Note that the pure FSO system refers to the use of FSO link from terrestrial to deep space detector, i.e., source-destination (S-D), while the hybrid system refers to source-relay-destination (S-R-D). Figure 7 illustrates average BER versus the average SNR under different scintillation indices. When the scintillation index is fixed, the average BER of the hybrid system is significantly lower than that of the FSO system; as the scintillation index increases, the intensity of the scintillation ascends, and the BER of the two systems increases accordingly. In addition, when $m^{2}<0.3$, the variation range of BER caused by the scintillation index is less than that when $m^{2}>0.3$. For example, in the $\mathrm{RF} / \mathrm{FSO}$ systems, when the SNR is $28 \mathrm{~dB}$, the difference of the BER is between $m^{2}=0.1$ and 0.3 is $5.5 \times 10^{-5}$, and between $m^{2}=0.5$ and 0.3 is $2.25 \times 10^{-4}$. It can be seen that the impact of increasing scintillation on BER performance may become more intense. Rytov theory is only aimed at the weak scintillation region, and the feature of the strong scintillation area needs to be studied in further research.

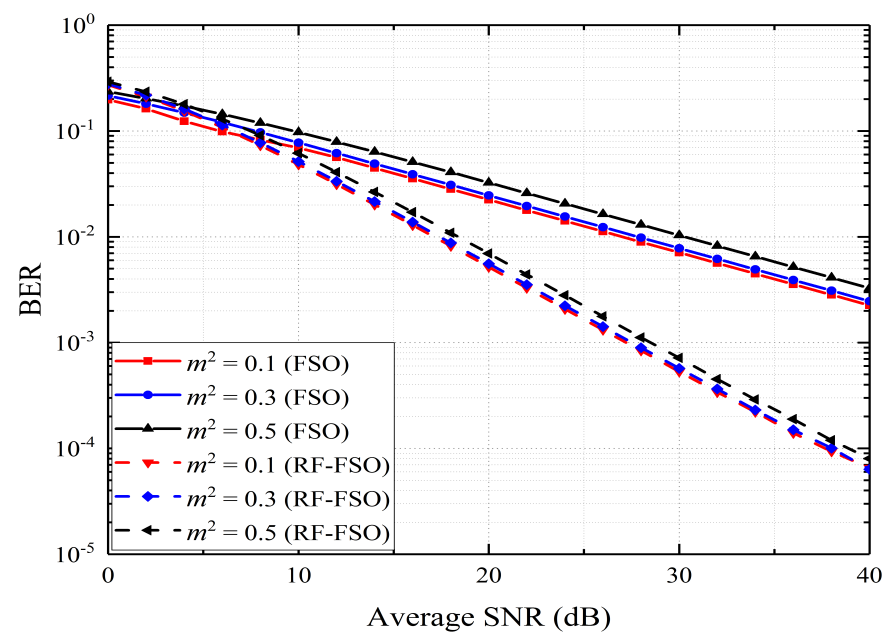

Figure 7. Average BER of 16-PPM-BPSK-SIM RF/FSO system and the FSO system against the average SNR under various scintillation indices and the pointing error parameter $\xi=1$.

Figure 8 illustrates the effect of the modulation technology on the average BER of the system and here we set the scintillation index as 0.3. Figure 8a shows the average BER of the hybrid RF/FSO system under three modulation modes. When $L>8$, the average BER 
of the hybrid modulation is lower than that of BPSK, and the average BER decrease as $L$ increases. The average BER performance of the PPM modulation in the hybrid system is still the best among these three modulation methods. However, considering other factors, such as the power efficiency of the L-PPM, the hybrid modulation is also worth studying. Figure $8 \mathrm{~b}$ compares the BER performance of the hybrid RF/FSO system (S-R-D) and pure FSO system (S-D) for L-PPM-BPSK-SIM modulation. The average BER of the hybrid system is significantly reduced, and when the average SNR reaches $20 \mathrm{~dB}$, the average BER of the hybrid system at $L=8$ is even lower than that of the FSO system at $L=64$. Combining Figure 8a,b, considering that an excessively large value of $L$ may reduce the communication rate, setting $L$ at 16 will be a decent option.

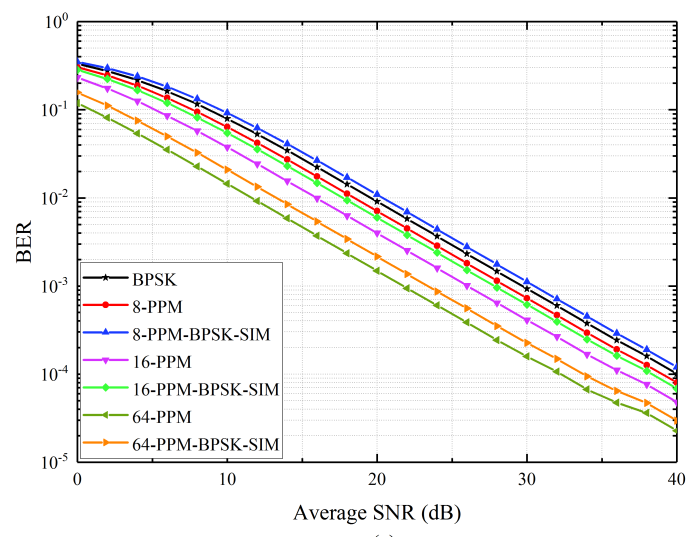

(a)

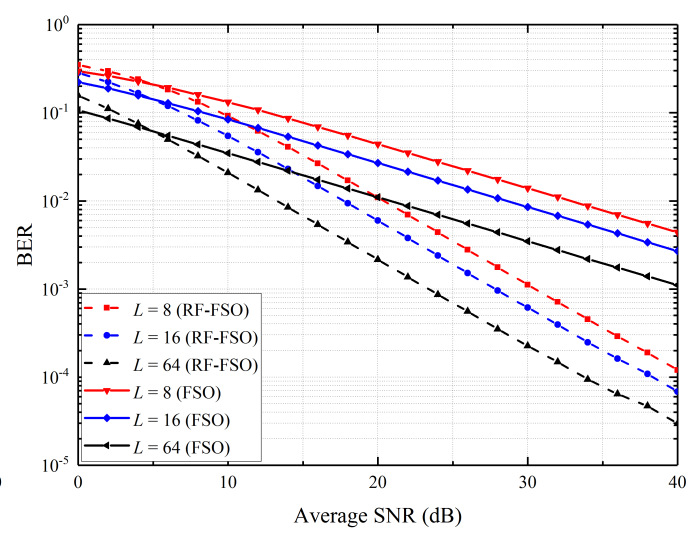

(b)

Figure 8. When $m^{2}=0.3$, i.e., under weak scintillation (a) average BER of BPSK, L-PPM and L-PPMBPSK-SIM against average SNR; (b) average BER of $L$-PPM-BPSK-SIM with various $L$ on the hybrid RF/FSO system and FSO system against the average SNR.

Figure 9 reveals the average BER performance of the hybrid RF/FSO system for optical wave propagation through coronal turbulence under various parameters. As shown in Figure 9a,b, the increasement of the turbulent outer scale and spectral index will lead to the reducement the average BER of the system. Moreover, when the values of $l_{0}$ and $p$ are larger, their influence on the BER will attenuate, because the amplitude fluctuation variance is smaller. However, it is worth noting that compared with an FSO system in some other research studies [41], when the turbulence outer scale and spectral index increase, the BER of the system will change significantly. Consequently, hybrid systems and hybrid modulation will enhance the stability of the BER performance.

Considering the effect of the wavelength on the scintillation index in Figure 3, the average BER versus wavelength is further studied in Figure 9c. As expected, the reduction of the wavelength improves the BER performance, so that the communication achieves better BER even with a low SNR. Therefore, decreasing the wavelength or increasing the carrier frequency are alternative methods to eliminate the coronal turbulence. As shown in Figure $9 \mathrm{~d}$, the relative solar wind density fluctuation factor also has an impact on the BER. As $\eta$ increases, the average BER of the system increases, correspondingly. Usually, we set $\eta$ within 0.2 because excessively large values of $\eta$ may contribute to an abnormal increase of the radio Sun. Here, to investigate the effect of $\eta$ on the BER more intuitively, we set the maximum value of $\eta$ at 0.4 . 

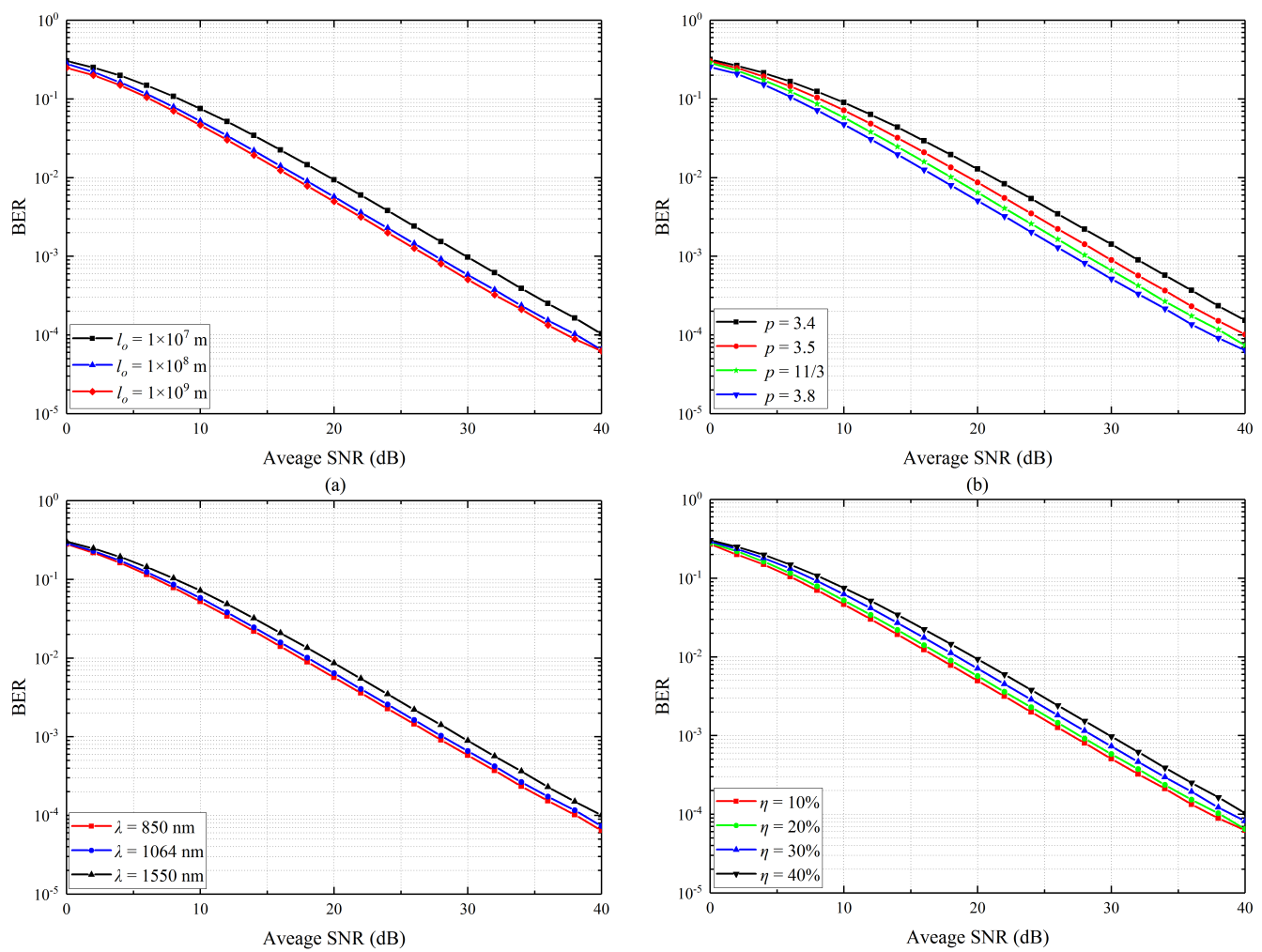

(c)

(d)

Figure 9. Under coronal turbulence, the average BER performance of the hybrid RF/FSO system against (a) outer scale, (b) spectral index, (c) wavelength, (d) density fluctuation factors.

Figure 10 describes the relationship between the average channel capacity and SNR. Here we simply consider the effects of the scintillation index and pointing error. As shown in Figure 10a, the average channel capacity decreases with the increase of the scintillation index. The reason is that solar scintillation leads to irregular fluctuation of signal amplitude, which produces changes in the power of the received signal. The pointing error also has a certain impact on the average channel capacity. In Figure 10b, when the scintillation index is fixed, the larger the pointing error parameter is, the larger the system capacity will be. For example, for SNR $=15 \mathrm{~dB}, m^{2}=0.3$, when $\xi=1$, the average capacity is 3.59 , and when $\xi=10$, the average capacity is 4.22 . Furthermore, the average channel capacity performance also follows the tendency that the larger the pointing error parameter, the smaller the impact on the performance of the system.

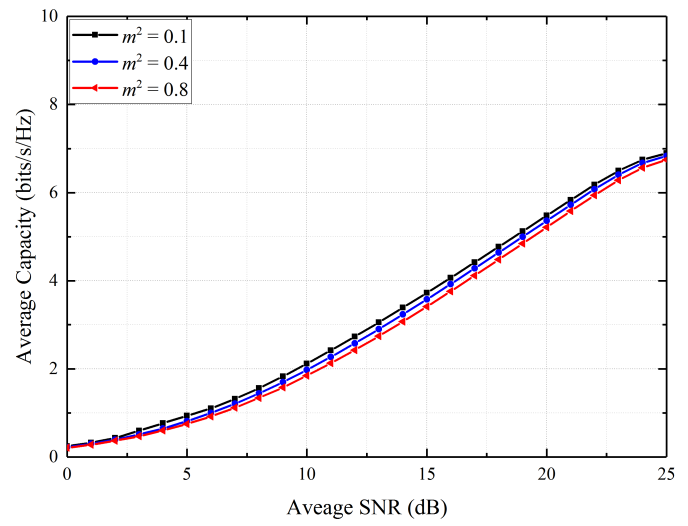

(a)

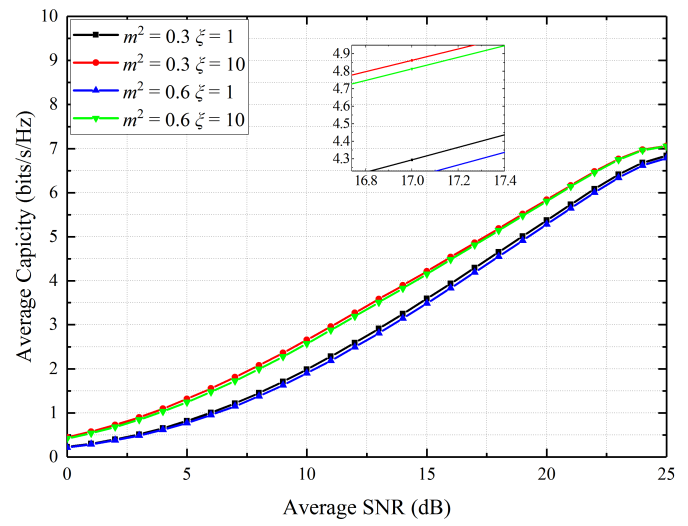

Figure 10. Average channel capacity against average SNR per hop (a) for a different scintillation index; (b) for different pointing error parameters. 


\section{Conclusions}

In this paper, we investigated the closed-form expression of the outage probability, average BER, and average channel capacity of the asymmetric dual-hop terrestrial-deep space hybrid RF/FSO system with a hybrid L-PPM-BPSK-SIM scheme. The simulation results illustrate that in deep space communication, the BER of the hybrid RF/FSO system is dozens of times lower than that of the FSO system. Hybrid modulation can improve the BER performance of the system, and the larger $L$ is, the greater the impact will be. Under the premise of ensuring the communication rate, the BER performance can be improved by increasing $L$. The small non-Kolmogorov spectral index, small solar wind density fluctuation factor, and large outer scale can reduce the BER of the system; reducing the wavelength is also a feasible method. In addition, we studied the effects of the scintillation index and pointing error on outage probability and average channel capacity. The larger the scintillation index and pointing error, the worse the system's channel capacity and outage probability performance.

Considering the practical technology, using a hybrid RF/ FSO system, reducing optical wavelength, decreasing pointing errors, and excellent modulation technology are options to mitigate the impact of solar scintillation in coronal turbulence and ultimately improve deep space communications performance. In future research, if more influencing factors are considered, such as background noise and thermal noise, more optimization techniques are needed [42]. Coding technologies, such as LDPC code [43], and a high-performance detector, such as a superconducting nanowire single photon detector (SNSPD) [44], are attracting more attention at present. In addition, the development of a cognitive deep space optical communication network and adaptive optical systems provide strong support to further improve deep space exploration [45]. Therefore, this study may provide a theoretical foundation and design basis for the future hybrid RF/FSO system in deep space exploration.

Author Contributions: Conceptualization, methodology, M.J. and H.L.; software, validation, writingoriginal draft preparation, M.J.; formal analysis, investigation, data curation, R.W., Z.W. and D.D.; writing-review and editing, visualization, Y.H. and W.L.; supervision, project administration, funding acquisition, H.L. All authors have read and agreed to the published version of the manuscript.

Funding: This work was supported in part by the National Natural Science Foundation of China (61875057, 62175070, 61774062); the Science and Technology Program of Guangzhou (2019050001), and the Natural Science Foundation of Guangdong (2021A1515012652).

Institutional Review Board Statement: Not applicable.

Informed Consent Statement: Not applicable.

Data Availability Statement: Not applicable.

Conflicts of Interest: The authors declare no conflict of interest.

\section{Appendix A}

We assumed that the subcarrier intensity modulation (SIM) is used in the relay $R$, the re-transmitted optical signal at this relay is given as [46-48]:

$$
r_{1}=\alpha_{1} s+n_{1}
$$

where $\alpha_{1}$ the is the fading amplitude of the Rayleigh fading channel for the S-R link, and the $n_{1}$ is an additive white Gaussian noise (AWGN) with the power spectral density of $N_{01}$. The $s$ indicates the RF signal transmitted from the source $S$.

Thereafter, the optical signal is forwarded from $\mathrm{R}$ to $\mathrm{D}$ via the FSO channel. At the destination, the received optical signal is as follows:

$$
r_{2}=A_{0} I\left\{G\left[1+\eta\left(\alpha_{1} s+n_{1}\right)\right]\right\}+n_{2}
$$

where $A_{0}$ is a constant for propagation. $G$ is the fixed relay gain at the relay $R$, and $\eta$ is the electrical-to-optical conversion coefficient. $I$ is a stationary random variable following a 
Gamma-Gamma distribution for the FSO link, and the $n_{2}$ is an AWGN with the power spectral density of $N_{02}$.

The overall signal-to-noise ratio (SNR) at the destination is as follows [9]:

$$
\gamma=\frac{I^{2} G^{2} \eta^{2} \alpha_{1}^{2} P_{1}}{I^{2} G^{2} \eta^{2} \alpha_{1}^{2} N_{01}+N_{02}}=\frac{\frac{\alpha_{1}^{2} P_{1}}{N_{01}} \frac{\eta^{2} I^{2}}{N_{02}}}{\frac{\eta^{2} I^{2}}{N_{02}}+\frac{1}{G^{2} N_{01}}},
$$

where the $P_{1}$ is the power transmitted at the source. Here, we consider the relay as a fixed gain, regardless of the fading information on the first link, we can let $C=\left(G^{2} N_{01}\right)^{-1}$. Therefore, the SNR can be rewritten as:

$$
\gamma=\frac{\gamma_{1} \gamma_{2}}{\gamma_{2}+C}
$$

\section{References}

1. Kaushal, H.; Kaddoum, G. Optical Communication in Space: Challenges and Mitigation Techniques. IEEE Commun. Surv. Tutor. 2017, 19, 57-96. [CrossRef]

2. Hemmati, H.; Biswas, A.; Djordjevic, I.B. Deep-Space Optical Communications: Future Perspectives and Applications. Proc. IEEE 2011, 99, 2020-2039. [CrossRef]

3. Xu, G.; Song, Z. Effects of Solar Scintillation on Deep Space Communications: Challenges and Prediction Techniques. IEEE Wirel. Commun. 2019, 26, 10-16. [CrossRef]

4. Mosavi, N.; Sequeira, H.B.; Copeland, D.J.; Menyuk, C.R. Solar scintillation study during planetary conjunction. In Proceedings of the 2016 IEEE Aerospace Conference, Big Sky, MT, USA, 5-12 March 2016; pp. 1-7. [CrossRef]

5. $\quad$ Efimov, A.I.; Lukanina, L.A.; Samoznaev, L.N.; Rudash, V.K.; Chashei, I.V.; Bird, M.K.; Pätzold, M.; MEX-VEX-ROS Radio Science Team. Two-Way Frequency Fluctuations Observed During Coronal Radio Sounding Experiments. Sol. Phys. 2014, 289, 1715-1729. [CrossRef]

6. Li, Q.; Yin, L.; Lu, J. Performance Study of A Deep Space Communications System with Low-Density Parity-Check Coding under Solar Scintillation. Int. J. Commun. 2012, 6, 1-9.

7. Xu, G.; Song, Z. Amplitude fluctuations for optical waves propagation through non-Kolmogorov coronal solar wind turbulence channels. Opt. Express 2018, 26, 8566-8580. [CrossRef]

8. $\mathrm{Xu}, \mathrm{G}$. Error performance of deep space optical communication with M-ary pulse position modulation over coronal turbulence channels. Opt. Express 2019, 27, 13344-13356. [CrossRef]

9. Eunju, L.; Park, J.; Han, D.; Yoon, G. Performance Analysis of the Asymmetric Dual-Hop Relay Transmission with Mixed RF/FSO Links. IEEE Photon. Technol. Lett. 2011, 23, 1642-1644. [CrossRef]

10. Bhatnagar, S.A.; Bhatnagar, M.R. Performance of an Amplify-and-Forward Dual-Hop Asymmetric RF-FSO Communication System. J. Opt. Commun. Netw. 2015, 7, 124-135. [CrossRef]

11. Ansari, I.S.; Yilmaz, F.; Alouini, M. Impact of Pointing Errors on the Performance of Mixed RF/FSO Dual-Hop Transmission Systems. IEEE Wirel. Commun. Lett. 2013, 2, 351-354. [CrossRef]

12. Matricciani, E. Deep-space communications with a 2-hop downlink with high link-availability. Int. J. Satell. Commun. Netw. 2005, 23, 203-228. [CrossRef]

13. Matricciani, E. An optimum design of deep-space downlinks affected by tropospheric attenuation. Int. J. Satell. Commun. Netw. 2009, 27, 312-329. [CrossRef]

14. Vilnrotter, V.; Hoppe, D.; Moision, B.; Charles, J. Optical communications performance of the hybrid 34-meter microwave antennas. In Proceedings of the 2010 IEEE Aerospace Conference, Big Sky, MT, USA, 6-13 March 2010; pp. 1-13. [CrossRef]

15. Cesarone, R.J.; Abraham, D.S.; Shambayati, S.; Rush, J. Deep-space optical communications. In Proceedings of the ICSOS, Santa Monica, CA, USA, 11-13 May 2011; pp. 410-423. [CrossRef]

16. Xu, G.; Zheng, Z.; Wang, W. Dual-hop deep space-terrestrial FSO/RF communication under solar scintillation: Performance analysis and challenges. China Commun. 2020, 17, 27-37. [CrossRef]

17. Liu, H.; Jiang, T.; Hao, Y. Research on improving deep space communication using hybrid RF-FSO system. J. Commun. 2020, 41, 148-155. [CrossRef]

18. Ho, C.; Wheelon, A. Amplitude Scintillation due to Atmospheric Turbulence for the Deep Space Network Ka-Band Downlink. IPN Prog. Rep. 2004, 42, 158.

19. Efimov, A.; Armand, N.; Lukanina, L.; Samoznaev, L.; Chashei, I.; Bird, M.; Plettemeier, D. Radial dependence of the level of amplitude fluctuations of spacecraft radio signals probing circumsolar plasma. J. Commun. Technol. Electron. 2008, 53, 1186-1194. [CrossRef]

20. Xu, G.; Song, Z. Solar Scintillation Effects on the Deep Space Communication Performance for Radio Wave Propagation Through Non-Kolmogorov Turbulence. IEEE Antennas Wirel. Propag. Lett. 2018, 17, 1505-1509. [CrossRef]

21. Gajić, A.; Radovanović, J.; Vuković, N.; Milanović, V.; Boiko, D.L. Theoretical approach to quantum cascade micro-laser broadband multimode emission in strong magnetic fields. Phys. Lett. A 2021, 387, 127007. [CrossRef] 
22. Garlinska, M.; Pregowska, A.; Masztalerz, K.; Osial, M. From mirrors to free-space optical communication-Historical aspects in data transmission. Future Internet 2020, 12, 179. [CrossRef]

23. Lionis, A.; Peppas, K.; Nistazakis, H.E.; Tsigopoulos, A.D.; Cohn, K. Experimental performance analysis of an optical communication channel over maritime environment. Electronics 2020, 9, 1109. [CrossRef]

24. Wheelon, A.D. Electromagnetic Scintillation. Vol. 1: Geometrical Optics; Cambridge University Press: Cambridge, UK, 2001. [CrossRef]

25. Thejappa, G.; Macdowall, R. Effects of Scattering on Radio Emission from the Quiet Sun at Low Frequencies. Astrophys. J. 2008, 676, 1338-1345. [CrossRef]

26. Calves, G.; Pogrebenko, S.; Cimo, G.; Duev, D.; Bocanegra-Bahamón, T.; Wagner, J.; Kallunki, J.; Vicente, P.; Kronschnabl, G.; Haas, R.; et al. Observations and analysis of phase scintillation of spacecraft signal on the interplanetary plasma. Astron. Astrophys. 2014, 564, A4. [CrossRef]

27. Ho, C.; Morabito, D.; Woo, R. Solar corona effects on angle of arrival fluctuations for microwave telecommunication links during superior solar conjunction. Radio Sci. 2008, 43, 1-13. [CrossRef]

28. Wing, S.; Johnson, J.; Chaston, C.; Echim, M.; Escoubet, C.; Lavraud, B.; Lemon, C.; Nykyri, K.; Otto, A.; Raeder, J.; et al. Review of Solar Wind Entry into and Transport Within the Plasma Sheet. Space Sci. Rev. 2014, 184, 33-86. [CrossRef]

29. Wheelon, A.D. Electromagnetic Scintillation. Vol. 2: Weak Scattering; Cambridge University Press: Cambridge, UK, 2003. [CrossRef]

30. Gradshteyn, I.; Ryzhik, I. Table of Integrals, Series and Products; American Mathematical Society: Providence, RI, USA, 2014; Volume 36. [CrossRef]

31. Al-Habash, M.; Andrews, L.; Philips, R. Mathematical model for the irradiance PDF of a laser beam propagating through turbulent media. Opt. Eng. 2001, 40, 1554-1562. [CrossRef]

32. Lee, L.; Jokipii, J. Strong scintillations in astrophysics. III-The fluctuations in intensity. Astrophys. J. 1975, 202, 439-453. [CrossRef]

33. Liu, H.; Liao, R.; Wei, Z.; Hou, Z.; Qiao, Y. Performance Improvement for Mixed RF-FSO Communication System by Adopting Hybrid Subcarrier Intensity Modulation. Appl. Sci. 2019, 9, 3724. [CrossRef]

34. Liu, H.; Liao, R.; Wei, Z.; Hou, Z.; Qiao, Y. BER Analysis of a Hybrid Modulation Scheme Based on PPM and MSK Subcarrier Intensity Modulation. IEEE Photon. J. 2015, 7, 1-10. [CrossRef]

35. Elganimi, T. Performance Comparison between OOK, PPM and PAM Modulation Schemes for Free Space Optical (FSO) Communication Systems: Analytical Study. Int. J. Comput. Appl. 2013, 79, 22-27. [CrossRef]

36. Faridzadeh, M.; Gholami, A.; Rajbhandari, S. Hybrid pulse position modulation and binary phase shift keying subcarrier intensity modulation for free space optics in a weak and saturated turbulence channel. J. Opt. Soc. Am. A 2012, 29, 1680-1685. [CrossRef]

37. Si, C.; Zhang, Y.; Wang, Y.; Wang, J.; Jia, J. Average capacity for non-Kolmogorov turbulent slant optical links with beam wander corrected and pointing errors. Optik 2012, 123, 1-5. [CrossRef]

38. Ansari, I.; Al-ahmadi, S.; Yilmaz, F.; Alouini, M.S. A New Formula for the BER of Binary Modulations with Dual-Branch Selection over Generalized-K Composite Fading Channels. IEEE Trans. Wirel. Commun. 2011, 59, 2654-2658. [CrossRef]

39. Raible, D.E.; Romanofsky, R.R.; Budinger, J.M.; Nappier, J.; Hylton, A.; Swank, A.J.; Nerone, A.L. On the physical realizability of the hybrid RF and optical communications platforms for deep space applications. In Proceedings of the 32nd ICSSC, San Diego, CA, 4-7 August 2014; p. 4482. [CrossRef]

40. Miridakis, N.; Matthaiou, M.; Karagiannidis, G. Multiuser relaying over mixed RF/FSO links. IEEE Trans. Wirel. Commun. 2014, 62, 1634-1645. [CrossRef]

41. Xu, G.; Zeng, M. Solar scintillation effect for optical waves propagating through Gamma-Gamma coronal turbulence channels IEEE Photon. J. 2019, 11, 1-15. doi:10.1109/JPHOT .2019.2924039. [CrossRef]

42. Ivanov, H.; Leitgeb, E.; Plank, T.; Kraus, D. Evaluation of FSO-technology as a candidate for reliable long-distance communication links for deep space applications. In Proceedings of the PIERS-Spring, Rome, Italy, 17-20 June 2019; pp. 3441-3448. [CrossRef]

43. Hormsup, K.; Imtawil, V.; Suthisopapan, P. Rate-compatible shortened LDPC codes for deep space fading channel. J. Commun. 2019, 14, 574-579. [CrossRef]

44. Li, B.; Liu, Y.; Tong, S.; Zhang, L.; Yao, H. BER analysis of a deep space optical communication system based on SNSPD over double generalized Gamma channel. IEEE Photonics J. 2018, 10, 1-7. [CrossRef]

45. Wang, Y.; Xu, H.; Li, D.; Wang, R.; Jin, C.; Yin, X.; Gao, S.; Mu, Q.; Xuan, L.; Cao, Z. Performance analysis of an adaptive optics system for free-space optics communication through atmospheric turbulence. Sci. Rep. 2018, 8, 1124. [CrossRef]

46. Peppas, K.P.; Datsikas, C.K. Average symbol error probability of general-order rectangular quadrature amplitude modulation of optical wireless communication systems over atmospheric turbulence channels. J. Opt. Commun. Netw. 2010, 2, 102-110. [CrossRef]

47. Popoola, W.O.; Ghassemlooy, Z. BPSK subcarrier intensity modulated free-space optical communications in atmospheric turbulence. J. Lightw. Technol. 2009, 27, 967-973. [CrossRef]

48. Park, J.; Lee, E.; Yoon, G. Average bit-error rate of the Alamouti scheme in Gamma-Gamma fading channels. IEEE Photon. Technol. Lett. 2010, 23, 269-271. [CrossRef] 